\title{
Supramolecular polymers
}

Tom F. A. de Greef and E. W. Meijer

\section{Most polymers consist of long molecular chains made up of many units connected by covalent bonds - but supramolecular polymers are different. The strikingly dynamic properties of these materials arise from the reversible bonds that hold their chains together, and open up the prospect of many new applications.}

\section{Do we need another class of polymer?}

In short, yes. Conventional polymers have excellent properties as materials, but when they melt they become highly viscous - the result of entanglement of their macromolecules. High temperatures and pressures are typically required to provide a melt of sufficiently low viscosity for processing, and this limits their applications. But supramolecular polymers combine good material properties with lowviscosity melts that are easy to handle. Some supramolecular polymers also have remarkable characteristics unique to their class, such as the ability to self-heal fractures in their structure.

\section{What exactly are supramolecular polymers?}

They are polymeric arrays of monomer units, held together by reversible and highly directional secondary interactions - that is, noncovalent bonds, such as hydrogen bonds. The resulting materials therefore maintain their polymeric properties in solution. The directions and strengths of the interactions are precisely tuned so that the array of molecules behaves as a polymer (that is, it behaves in a way that can be described by the theories of polymer physics). The high reversibility of the non-covalent bonds ensures that supramolecular polymers are always formed under conditions of thermodynamic equilibrium, and hence the lengths of the chains are directly related to the strength of the non-covalent bond, the concentration of the monomer and the temperature.

\section{How do they differ from conventional polymers?}

Most, if not all, of the material properties of conventional polymers stem from the covalent nature of their spaghetti-like molecules, although secondary interactions between the molecules are also often involved. For example, hydrogen bonding in nylon affects the properties of the polymer's crystals. Supramolecular polymers, on the other hand, make use of reversible interactions in the main chain. Hence, all the mechanical properties of supramolecular polymers are a direct result of secondary interactions, in particular the strength, reversibility and directionality of these interactions.

\section{What are the origins of supramolecular polymers?}

Supramolecular interactions between certain molecules have long been recognized. In most cases, only short chains of up to about 10 units are created, whereas useful supramolecular polymers require at least 100 to 1,000 monomers to be connected at any one time. The key to creating longer chains is to strengthen the interactions between the monomers; put more accurately, the average lifetime of the bonds must be increased. But the window of opportunity is small - if the secondary interactions are too strong, the polymers lose their dynamic flexibility, and their bulk properties become more like those of covalent polymers.

\section{What kick-started the field?}

Perhaps the most crucial step was the development in 1997 of a unit that could form four hydrogen bonds, known as 2-ureido-4[1H]pyrimidinone (UPy). A monomer containing two of these self-complementary units self-assembles into polymers (Fig. 1). The relatively long lifetime of the bonds $(0.1-1 \mathrm{sec}-$ onds) was essential for making networks that
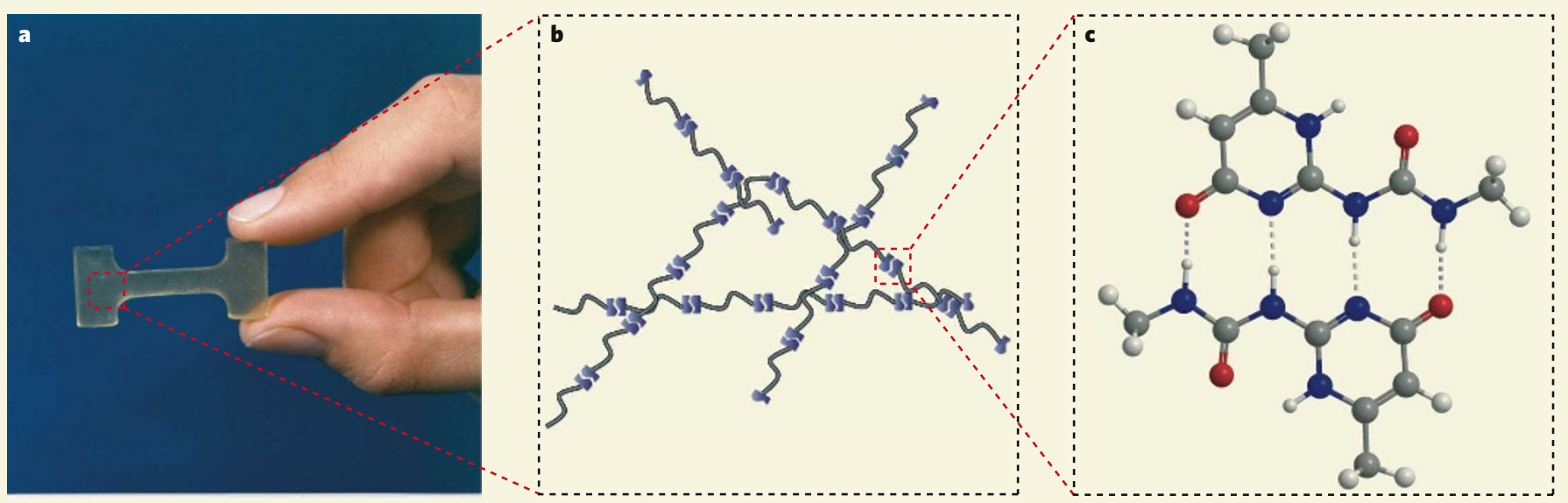

Figure $1 \mid$ Zooming in on supramolecular polymers. a, This plastic component is made from a supramolecular polymer. $\mathbf{b}$, The molecular structure is a network of monomers connected by hydrogen bonds. Each monomer consists of a hydrocarbon chain with a hydrogen-bonding unit
(2-ureido-4[1H]-pyrimidinone, UPy, in blue) at each end. c, The UPy units form hydrogen bonds to each other (dotted lines). The monomers therefore spontaneously self-assemble to form the polymeric network. Carbon atoms are shown in grey; nitrogen in blue; oxygen in red; and hydrogen in white. 


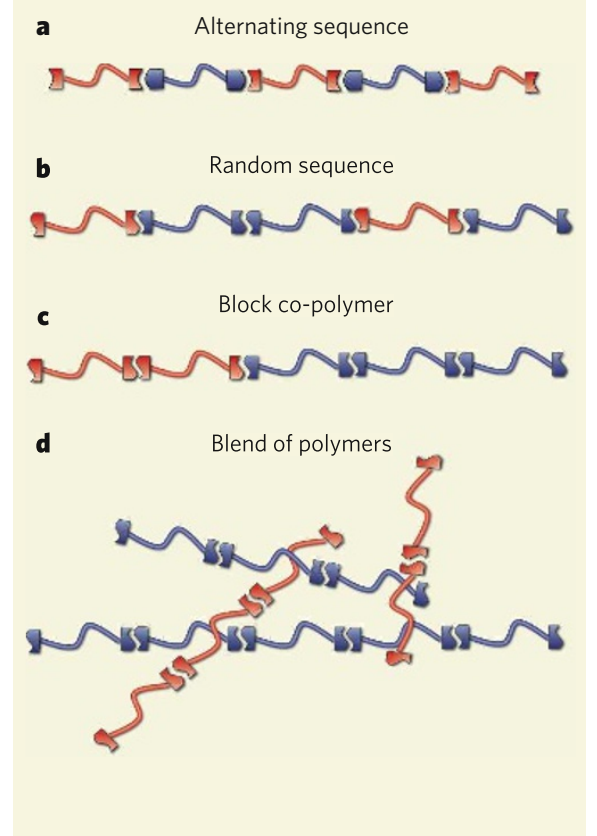

Figure 2 | Monomer ordering in supramolecular co-polymers. These co-polymers form from mixtures of different kinds of self-assembling monomer. The ordering of the monomers depends on the binding modes of their hydrogen-bonding groups. a, If the different kinds of group on each monomer form complementary bonds (only to each other), an alternating sequence of monomer types forms. b-d, If the groups are self-complementary (they bind to their own kind) but are also able to form hydrogen bonds to other kinds of group, there are three different possibilities. b, Normally, a random chain of monomers forms.

c, Alternatively, a chain assembles containing distinct blocks of one kind of monomer. This can happen when, for example, there are two self-complementary arrays $\mathrm{AA}$ and $\mathrm{BB}$ that can form a self-complementary $A B$ interaction of much lower strength. $d$, If the monomers repel each other and don't mix, the polymer forms as a blend of chains that each contain one kind of monomer.

displayed true polymeric properties. Before the discovery of UPy, supramolecular polymers were based on pairs of different monomers that formed complementary bonds with each other - but even a slight excess of one of the monomers greatly reduced the degree of polymerization.

\section{Are hydrogen bonds the best way to hold supramolecular polymers together?}

Hydrogen bonds are ideal, as they combine high strength with excellent reversibility. Furthermore, because they form bonds only in one direction (that of the polymer chain), unwanted interactions in other directions are minimized. The strength and reversibility of hydrogen bonding between monomers can be precisely tuned by installing an array of hydrogen-bonding groups in the monomers, so allowing several bonds to form. The interactions between such strongly bound units are maintained even when the polymer is dissolved in water, where the solvent molecules compete to form hydrogen bonds with the monomers. Nevertheless, in water, the hydrogen bonding between monomers is much weaker than in the solid material. In order to make supramolecular polymers of sufficient length in water, the monomers must form hydrophobic pockets in which hydrogen bonds are protected from the detrimental influence of the solvent.

\section{Are supramolecular polymers easy to make?}

Because the polymers self-assemble from monomers (Box 1), the real question is whether the monomers can be easily made. The answer is that it depends on the monomer - some require several steps of sophisticated organic synthesis, but others are easy to make, even on a large scale. Luckily, most of the interesting materials fall into the second category. For instance, the UPy unit is made in one step from commercially available compounds. Furthermore, the monomers for supramolecular polymers can be purified using the plethora of methods that have been developed for small organic molecules. This is an advantage over covalent polymers, which are more difficult to purify.

\section{Can two or more kinds of monomer be combined?}

Yes, simply by mixing different monomers together. The properties of the resulting supramolecular co-polymers can be easily tuned in a modular approach by changing the ratio of the monomers. For co-polymers with an alternating sequence of units, the two different monomers should have complementary binding motifs whereas, for random sequences, self-complementary monomers are preferred. But if the monomers strongly repel each other (for example, by hydrophobic interactions), they might not form mixed chains, so that the resulting material actually forms as a blend of two different polymers (Fig. 2). The modular approach to making supramolecular co-polymers is particularly useful for adjusting the properties of materials for medical applications, such as tissue engineering and drug delivery. These materials must be fine-tuned to meet the exact needs of biological systems.

\section{What is the chief selling point of supramolecular polymers?}

Perhaps their biggest advantage is the strong dependence of their melt viscosities on temperature - above the material's melting point (or glass transition temperature), small increases in temperature lead to a large reduction in viscosity. This allows the materials to be easily processed, and will be useful for several applications. For example, inks in ink-jet printers need to be runny when they're squirted out of the pen, but must become almost solid when they hit the paper. The properties of supramolecular polymers fit the bill well.

\section{Why do the material properties depend so much on temperature?}

Because of the mechanisms of stress relaxation that are available to supramolecular polymers. The dominant pathway for stress relaxation in conventional polymers is reptation - a snakelike movement of the polymeric chains. Stress relaxation in supramolecular polymers also occurs by reptation, but additionally, supramolecular chains can release strain by breaking and then recombining with other, strain-free chain-ends. This last process is unique to supramolecular polymers, and speeds up at higher temperatures. Finally, the chains in these polymers rapidly become shorter with increasing temperature, leading to liquid-like behaviour.

\section{Can this temperature-dependent behaviour be modified?}

Certainly. Second-generation supramolecular polymers are designed to incorporate additional interactions - usually lateral hydrogen bonds - between groups at the ends of the polymer chains to strengthen the materials. These end-groups are usually attached to oligomers that have molecular masses of only a few thousand daltons; typical polymer chains have molecular masses of several hundred thousand daltons. In this way, supramolecular polymers have been made that melt at higher temperatures, but that are elastic at temperatures below their melting point. The enhanced material properties of these polymers are a direct result of lateral hydrogen bonding between the end-groups, which causes the material to segregate into long nanofibres. Further studies on this phenomenon will show whether this is a general effect.

\section{Are there other interesting properties?}

A supramolecular rubber was recently reported that can self-heal: fractures in the sample can be repaired simply by pressing the two broken edges of the material together. The inventors of the rubber propose that fractures occur when hydrogen bonds between monomers in the material are broken; bringing the edges of the fracture together allows the hydrogen bonds to re-form, sealing up the gap. Impressively, the dynamic behaviour of the hydrogen bonds - necessary for the self-healing process - does not compromise the properties of the material.

\section{Which applications are closest to the marketplace?}

Supramolecular polymers might find their first use as coatings for delicate, heat-sensitive substrates. For example, wood and fabrics 
Box 1 | Mechanisms of supramolecular polymerization
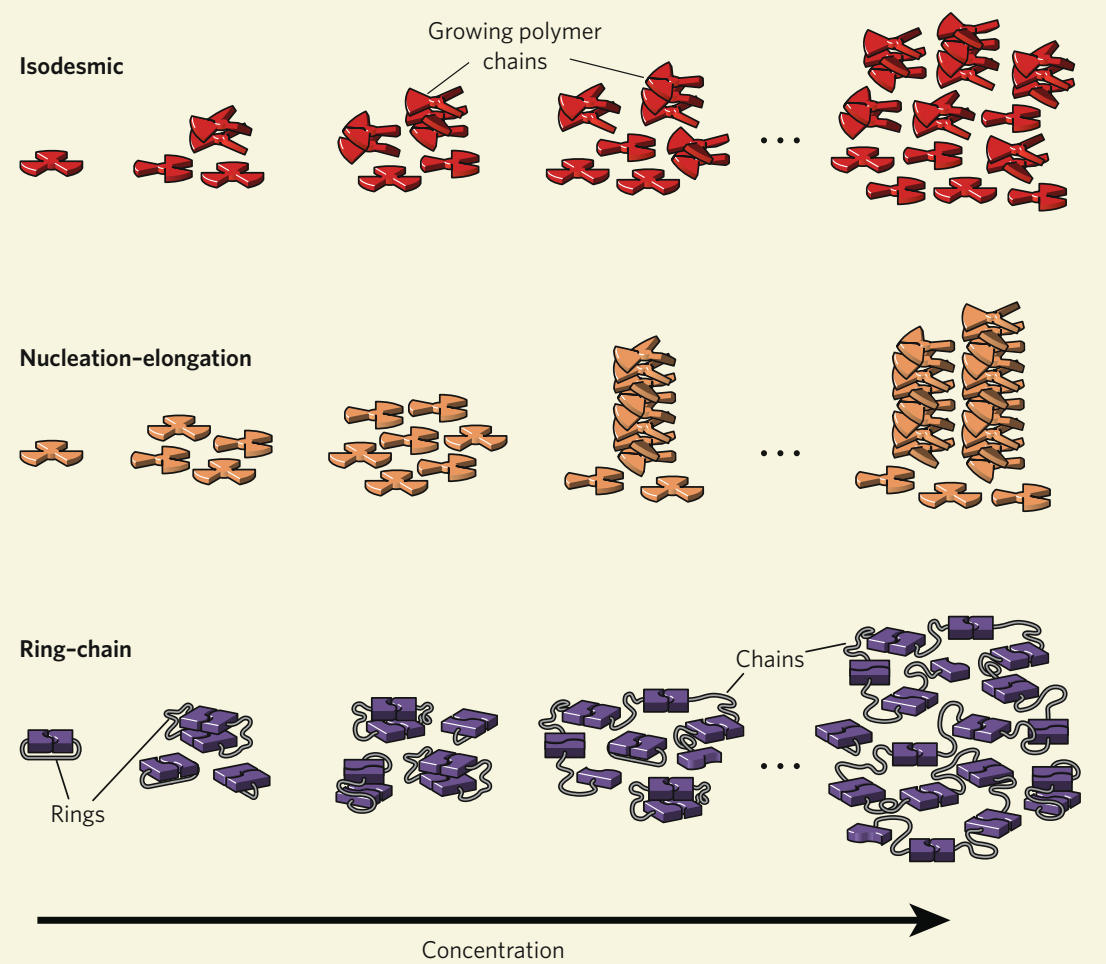

There are three main growth mechanisms for supramolecular polymerization. The first, known as isodesmic growth, occurs when the strength of secondary interactions between monomers in the polymer chains is unaffected by the length of the chain. Each monomer added to a growing chain therefore increases the thermodynamic stability of the system by the same amount. Because each additional step is equivalent, no critical temperature or concentration of monomers is required for polymerization to occur. Instead, the concentration of polymer chains rises gradually as the concentration of monomers in the solution is increased, or as the temperature increases.

The second process

\begin{tabular}{|c|c|}
\hline $\begin{array}{l}\text { - the nucleation-elongation } \\
\text { mechanism - occurs in } \\
\text { the growth of 'ordered' } \\
\text { supramolecular polymers, } \\
\text { such as those that form } \\
\text { helices. This involves two } \\
\text { distinct phases of self- } \\
\text { assembly: a slow nucleation } \\
\text { phase followed by a more } \\
\text { rapid growth phase. In this } \\
\text { mechanism, the non-covalent } \\
\text { bonds between monomers in } \\
\text { chains are weak, hindering the } \\
\text { initial formation of the chains. } \\
\text { But once a stable nucleus is } \\
\text { formed, further monomer } \\
\text { addition becomes favourable, } \\
\text { at which point polymer growth } \\
\text { accelerates. Long polymer } \\
\text { chains will form only above } \\
\text { a certain temperature and } \\
\text { minimum concentration of } \\
\text { monomer. } \\
\text { The third growth process } \\
\text { is known as the ring-chain } \\
\text { mechanism. This is a }\end{array}$ & $\begin{array}{l}\text { special case of nucleated } \\
\text { polymerization that also } \\
\text { occurs in covalent polymers; } \\
\text { it is characterized by an } \\
\text { equilibrium between closed } \\
\text { rings of monomers and linear } \\
\text { chains. In this mechanism, } \\
\text { below a certain monomer } \\
\text { concentration, the ends of } \\
\text { any small polymer chains } \\
\text { are more likely to encounter } \\
\text { and react with each other } \\
\text { (forming a ring) than they } \\
\text { are to find another monomer } \\
\text { that would result in chain } \\
\text { elongation. Above the } \\
\text { critical concentration, chain } \\
\text { formation becomes more } \\
\text { favourable and polymer } \\
\text { growth occurs rapidly. } \\
\text { As in the nucleation- } \\
\text { elongation mechanism, } \\
\text { the degree of polymerization } \\
\text { changes abruptly once } \\
\text { critical conditions are } \\
\text { reached. }\end{array}$ \\
\hline
\end{tabular}

can't usually be coated with covalent polymers because they would be damaged by the hot polymer melts. But this wouldn't be a problem for supramolecular polymers with their relatively low melting points. Supramolecular polymers can also be made into excellent adhesives, and their potential as inks was mentioned earlier. Finally, the materials are also attractive for any applications that require processing from a solution or a gel, such as cosmetics and personal-care products.

\section{Are there any naturally occurring supramolecular polymers?}

Yes, but not many. Some protein molecules form small networks in order to function, but don't make long enough chains to be classed as polymers. Others - such as G-actin and tubulin, which act as building-blocks for the scaffolding of cells - genuinely polymerize to form filaments, but the mechanism involves an irreversible reaction with phosphatecontaining 'fuel' molecules. The polymerization is therefore not an equilibrium process, allowing the resulting materials to display some exciting properties. For example, tubulin dimers form long, rigid polymers known as microtubules, which can synchronize their growth (or shrinkage). But the greatest resemblance to synthetic supramolecular polymerization is seen in the self-assembly of the tobacco mosaic virus, as the assembly process occurs without the use of fuel molecules.

\section{What will the future bring?}

Supramolecular polymers will become an integral part of the general field of self-assembling polymers, and will find applications in areas ranging from electronics to medicine. The complexity of their molecular systems will increase rapidly, leading in turn to the development of yet more unconventional polymeric properties. But the most intriguing challenge will be the search for self-organizing (rather than selfassembling) supramolecular polymers.

\section{What are self-organized polymers?}

These materials will also be formed from monomers using secondary interactions, but the polymerization will be far from an equilibrium process - in self-organized structures, external energy is required to maintain a steady state, along with an open system that allows a continuous inflow of monomers. In theory, such a material would display both spatial and temporal order, on a much larger length- and timescale than seen in structures formed under equilibrium conditions. Self-organized polymers will thus mimic the dynamic behaviour of proteins such as tubulin. The critical requirements for self-organizing supramolecular polymers are auto-catalytic reactions (in which the products of reactions between monomers catalyse further polymerization reactions), a supply of chemical energy, and processes that involve competing diffusion of the molecules involved in the polymerization. These requirements have yet to be met in synthetic systems. With so much work still to be done, there has never been a more exciting time to work with supramolecular polymers.

Tom F. A. de Greef and E. W. Meijer are in the Laboratory of Macromolecular and Organic Chemistry, Eindhoven University of Technology, PO Box 513, 5600 MB Eindhoven, the Netherlands. e-mails: t.f.a.d.greef@tue.nl; e.w.meijer@tue.nl

\section{FURTHER READING}

Brunsveld, L., Folmer, B. J. B., Meijer, E. W. \& Sijbesma, R. P. Chem. Rev. 101, 4071-4098 (2001).

Ciferri, A. (ed.) Supramolecular Polymers (CRC,

New York, 2005).

Lehn, J.-M. Polym. Int. 51, 825-839 (2002).

Binder, W. (ed.) Hydrogen Bonded Polymers (Springer, New York, 2007).

www.suprapolix.com/index.php?page $=$ supramolecular Dankers, P. Y. W. \& Meijer, E. W. Bull. Chem. Soc. Jpn 80, 2047-2073 (2007).

Cordier, P., Tournilhac, T., Soulié-Ziakovic, C. \& Leibler, L. Nature 451, 977-980 (2008).

Competing financial interests: declared (see online article for details). 